(C) 2006 Claus Gerhardt

Adv. Theor. Math. Phys. 10 (2006) 33-48

\title{
The mass of a Lorentzian manifold
}

\author{
Claus Gerhardt
}

Ruprecht-Karls-Universität, Institut Für Angewandte Mathematik, Im Neuenheimer Feld 294, 69120 Heidelberg, Germany

gerhardt@math.uni-heidelberg.de

\begin{abstract}
We define a physically reasonable mass for an asymptotically Robertson-Walker manifold which is uniquely defined in the case of a normalized representation.
\end{abstract}

\section{Contents}

1. Introduction

2. Notations and definitions

3. Proof of Theorem 1.3

4. Uniqueness of the mass

5. A variant of the Penrose inequality

6. An example

Acknowledgment

References

48

e-print archive: http://lanl.arXiv.org/abs/math.DG/0403002 


\section{Introduction}

For asymptotically flat Lorentzian 4-manifolds, the so-called ADM-mass is defined by looking at a space-like slice $M=\{t=$ const $\}$. If $M$ is asymptotically flat and the scalar curvature of $M$ of class $L^{1}(M)$, then the ADM-mass is defined as a flux integral

$$
m_{\mathrm{ADM}}=\lim _{r \rightarrow \infty} \frac{1}{16 \pi} \int_{\partial B_{r}(0)}\left(g_{i i, j}-g_{i j, i}\right) \nu^{j} ;
$$

the limit is finite iff $\int_{M}|R|<\infty$, cf. [1]. Schoen and Yau [6,7] proved that $m \geq 0$ if $R \geq 0$ with equality if and only if $M$ is isometric to Euclidean space.

If $M$ is an exterior region with compact boundary consisting of minimal surfaces, then the Penrose inequality states that

$$
16 \pi m^{2} \geq|C|
$$

where $|C|$ is the area of any connected component of $\partial M$. The Penrose inequality was proved by Huisken and Ilmanen [5].

For general Lorentzian manifolds, e.g., for cosmological spacetimes, there is no notion of mass as far as we know. We shall define a physically reasonable mass for asymptotically Robertson-Walker (ARW) spacetimes - see Definition 1.1 - that satisfy the time-like convergence condition

$$
\bar{R}_{\alpha \beta} \nu^{\alpha} \nu^{\beta} \geq 0 \quad \forall\langle\nu, \nu\rangle=-1 .
$$

In [3], we introduced the notion of ARW spacetimes and proved some convergence theorems for solutions to the inverse mean curvature flow (IMCF) in these spacetimes.

Definition 1.1. A cosmological spacetime $N$, $\operatorname{dim} N=n+1$, is said to be ARW with respect to the future, if a future end of $N, N_{+}$, can be written as a product $N_{+}=[a, b) \times \mathcal{S}_{0}$, where $\mathcal{S}_{0}$ is a compact Riemannian space, and there exists a future directed time function $\tau=x^{0}$ such that the metric in $N_{+}$can be written as

$$
d \bar{s}^{2}=\mathrm{e}^{2 \tilde{\psi}}\left\{-d x^{0^{2}}+\sigma_{i j}\left(x^{0}, x\right) d x^{i} d x^{j}\right\},
$$

where $\mathcal{S}_{0}$ corresponds to $x^{0}=a, \tilde{\psi}$ is of the form

$$
\tilde{\psi}\left(x^{0}, x\right)=f\left(x^{0}\right)+\psi\left(x^{0}, x\right),
$$

and we assume that there exists a positive constant $c_{0}$ and a smooth Riemannian metric $\bar{\sigma}_{i j}$ on $\mathcal{S}_{0}$ such that

$$
\lim _{\tau \rightarrow b} \mathrm{e}^{\psi}=c_{0} \wedge \lim _{\tau \rightarrow b} \sigma_{i j}(\tau, x)=\bar{\sigma}_{i j}(x),
$$


and

$$
\lim _{\tau \rightarrow b} f(\tau)=-\infty
$$

Without loss of generality, we shall assume $c_{0}=1$. Then $N$ is ARW with respect to the future, if the metric is close to the Robertson-Walker metric

$$
d \bar{s}^{2}=\mathrm{e}^{2 f}\left\{-d x^{0^{2}}+\bar{\sigma}_{i j}(x) d x^{i} d x^{j}\right\}
$$

near the singularity $\tau=b$. By close we mean that the derivatives of arbitrary order with respect to space and time of the conformal metric $e^{-2 f} \bar{g}_{\alpha \beta}$ in (1.4) should converge to the corresponding derivatives of the conformal limit metric in (1.8) when $x^{0}$ tends to $b$. We emphasize that in our terminology Robertson-Walker metric does not imply that $\left(\bar{\sigma}_{i j}\right)$ is a metric of constant curvature, it is only the spatial metric of a warped product.

We assume, furthermore, that $f$ satisfies the following five conditions

$$
-f^{\prime}>0
$$

there exists $\omega \in \mathbb{R}$ such that

$$
n+\omega-2>0 \wedge \lim _{\tau \rightarrow b}\left|f^{\prime}\right|^{2} \mathrm{e}^{(n+\omega-2) f}=m>0 .
$$

Set $\tilde{\gamma}=\frac{1}{2}(n+\omega-2)$, then there exists the limit

$$
\lim _{\tau \rightarrow b}\left(f^{\prime \prime}+\tilde{\gamma}\left|f^{\prime}\right|^{2}\right)
$$

and

as well as

$$
\left|D_{\tau}^{m}\left(f^{\prime \prime}+\tilde{\gamma}\left|f^{\prime}\right|^{2}\right)\right| \leq c_{m}\left|f^{\prime}\right|^{m} \quad \forall m \geq 1
$$

$$
\left|D_{\tau}^{m} f\right| \leq c_{m}\left|f^{\prime}\right|^{m} \quad \forall m \geq 1
$$

We call $N$ a normalized ARW spacetime, if

$$
\int_{\mathcal{S}_{0}} \sqrt{\operatorname{det} \bar{\sigma}_{i j}}=\left|S^{n}\right| \text {. }
$$

\section{Remark 1.2.}

(i) If these assumptions are satisfied, then the range of $\tau$ is finite, hence, we may - and shall - assume w.l.o.g. that $b=0$, i.e.,

$$
a<\tau<0 \text {. }
$$

(ii) Any ARW spacetime can be normalized as one easily checks. Without a normalization condition, the constant $m$ in (1.10) would not be defined uniquely as we shall see. It will later be identified with the mass of $N$.

(iii) In view of the assumptions on $f$, the mean curvature of the coordinate slices $M_{\tau}=\left\{x^{0}=\tau\right\}$ tends to $\infty$, if $\tau$ goes to zero. 
(iv) Similarly, one can define $N$ to be ARW with respect to the past. In this case, the singularity would lie in the past, correspond to $\tau=0$, and the mean curvature of the coordinate slices would tend to $-\infty$.

Our main result is as follows.

Theorem 1.3. Let $N$ be a $(n+1)$-dimensional normalized ARW spacetime with respect to the future that satisfies the time-like convergence condition. Then the future mass $m$ of $N$ is defined by

$$
\frac{1}{2} n(n-1)\left|S^{n}\right| m=\lim \int_{M} G_{\alpha \beta} \nu^{\alpha} \nu^{\beta} \mathrm{e}^{\omega f} \mathrm{e}^{\psi},
$$

where $G_{\alpha \beta}$ is the Einstein tensor, $\omega$ the constant that appears in the definition of $A R W$ spaces, and the closed space-like hypersurfaces $M$ converge to the future singularity such that, if they are written as graphs over $\mathcal{S}_{0}$, $M=$ graph $u, D u$ vanishes when the singularity is approached.

For normalized ARW spaces, the mass is defined independently of the time function $x^{0}, f, \psi$, and $\omega$.

\section{Notations and definitions}

The main objective of this section is to state the equations of Gauß, Codazzi, and Weingarten for space-like hypersurfaces $M$ in a $(n+1)$-dimensional Lorentzian manifold $N$. Geometric quantities in $N$ will be denoted by $\left(\bar{g}_{\alpha \beta}\right),\left(\bar{R}_{\alpha \beta \gamma \delta}\right)$, etc. and those in $M$ by $\left(g_{i j}\right),\left(R_{i j k l}\right)$, etc. Greek indices range from 0 to $n$ and Latin from 1 to $n$; the summation convention is always used. Generic coordinate systems in $N$ resp. $M$ will be denoted by $\left(x^{\alpha}\right)$ resp. $\left(\xi^{i}\right)$. Covariant differentiation will simply be indicated by indices; only in the case of possible ambiguity, they will be preceded by a semicolon, i.e., for a function $u$ in $N,\left(u_{\alpha}\right)$ will be the gradient and $\left(u_{\alpha \beta}\right)$ the Hessian, but, e.g., the covariant derivative of the curvature tensor will be abbreviated by $\bar{R}_{\alpha \beta \gamma \delta ; \epsilon}$. We also point out that

$$
\bar{R}_{\alpha \beta \gamma \delta ; i}=\bar{R}_{\alpha \beta \gamma \delta ; \epsilon} x_{i}^{\epsilon}
$$

with obvious generalizations to other quantities.

Let $M$ be a space-like hypersurface, i.e., the induced metric is Riemannian, with a differentiable normal $\nu$ which is time-like. 
In local coordinates, $\left(x^{\alpha}\right)$ and $\left(\xi^{i}\right)$, the geometric quantities of the spacelike hypersurface $M$ are connected through the following equations

$$
x_{i j}^{\alpha}=h_{i j} \nu^{\alpha}
$$

the so-called Gauß formula. Here, and also in the sequel, a covariant derivative is always a full tensor, i.e.,

$$
x_{i j}^{\alpha}=x_{, i j}^{\alpha}-\Gamma_{i j}^{k} x_{k}^{\alpha}+\bar{\Gamma}_{\beta \gamma}^{\alpha} x_{i}^{\beta} x_{j}^{\gamma} .
$$

The comma indicates ordinary partial derivatives.

In this implicit definition, the second fundamental form $\left(h_{i j}\right)$ is taken with respect to $\nu$.

The second equation is the Weingarten equation

$$
\nu_{i}^{\alpha}=h_{i}^{k} x_{k}^{\alpha}
$$

where we remember that $\nu_{i}^{\alpha}$ is a full tensor.

Finally, we have the Codazzi equation

$$
h_{i j ; k}-h_{i k ; j}=\bar{R}_{\alpha \beta \gamma \delta} \nu^{\alpha} x_{i}^{\beta} x_{j}^{\gamma} x_{k}^{\delta}
$$

and the Gauß equation

$$
R_{i j k l}=-\left\{h_{i k} h_{j l}-h_{i l} h_{j k}\right\}+\bar{R}_{\alpha \beta \gamma \delta} x_{i}^{\alpha} x_{j}^{\beta} x_{k}^{\gamma} x_{l}^{\delta} .
$$

Now, let us assume that $N$ is a globally hyperbolic Lorentzian manifold with a compact Cauchy surface. $N$ is then a topological product $I \times \mathcal{S}_{0}$, where $I$ is an open interval, $\mathcal{S}_{0}$ is a compact Riemannian manifold, and there exists a Gaussian coordinate system $\left(x^{\alpha}\right)$, such that the metric in $N$ has the form

$$
d \bar{s}_{N}^{2}=\mathrm{e}^{2 \psi}\left\{-d x^{0^{2}}+\sigma_{i j}\left(x^{0}, x\right) d x^{i} d x^{j}\right\}
$$

where $\sigma_{i j}$ is a Riemannian metric, $\psi$ a function on $N$, and $x$ an abbreviation for the space-like components $\left(x^{i}\right)$. We also assume that the coordinate system is future oriented, i.e., the time coordinate $x^{0}$ increases on future directed curves. Hence, the contravariant time-like vector $\left(\xi^{\alpha}\right)=(1,0, \ldots, 0)$ is future directed as is its covariant version $\left(\xi_{\alpha}\right)=\mathrm{e}^{2 \psi}(-1,0, \ldots, 0)$. 
Let $M=\operatorname{graph} u_{\left.\right|_{\mathcal{S}_{0}}}$ be a space-like hypersurface

$$
M=\left\{\left(x^{0}, x\right): x^{0}=u(x), x \in \mathcal{S}_{0}\right\},
$$

then the induced metric has the form

$$
g_{i j}=\mathrm{e}^{2 \psi}\left\{-u_{i} u_{j}+\sigma_{i j}\right\}
$$

where $\sigma_{i j}$ is evaluated at $(u, x)$, and its inverse $\left(g^{i j}\right)=\left(g_{i j}\right)^{-1}$ can be expressed as

$$
g^{i j}=\mathrm{e}^{-2 \psi}\left\{\sigma^{i j}+\frac{u^{i}}{v} \frac{u^{j}}{v}\right\}
$$

where $\left(\sigma^{i j}\right)=\left(\sigma_{i j}\right)^{-1}$ and

$$
\begin{aligned}
& u^{i}=\sigma^{i j} u_{j} \\
& v^{2}=1-\sigma^{i j} u_{i} u_{j} \equiv 1-|D u|^{2} .
\end{aligned}
$$

Hence, graph $u$ is space-like if and only if $|D u|<1$.

The covariant form of a normal vector of a graph looks like

$$
\left(\nu_{\alpha}\right)= \pm v^{-1} \mathrm{e}^{\psi}\left(1,-u_{i}\right)
$$

and the contravariant version is

$$
\left(\nu^{\alpha}\right)=\mp v^{-1} \mathrm{e}^{-\psi}\left(1, u^{i}\right) .
$$

Thus, we have

Remark 2.1. Let $M$ be space-like graph in a future oriented coordinate system. Then the contravariant future directed normal vector has the form

$$
\left(\nu^{\alpha}\right)=v^{-1} \mathrm{e}^{-\psi}\left(1, u^{i}\right)
$$

and the past directed

$$
\left(\nu^{\alpha}\right)=-v^{-1} \mathrm{e}^{-\psi}\left(1, u^{i}\right)
$$

In the Gauß formula (2.2), we are free to choose the future or past directed normal, but we stipulate that we always use the past directed normal for reasons that we have explained in [2, Section 2].

Look at the component $\alpha=0$ in (2.2) and obtain in view of (2.15)

$$
\mathrm{e}^{-\psi} v^{-1} h_{i j}=-u_{i j}-\bar{\Gamma}_{00}^{0} u_{i} u_{j}-\bar{\Gamma}_{0 j}^{0} u_{i}-\bar{\Gamma}_{0 i}^{0} u_{j}-\bar{\Gamma}_{i j}^{0} .
$$

Here, the covariant derivatives are taken with respect to the induced metric of $M$, and

$$
-\bar{\Gamma}_{i j}^{0}=\mathrm{e}^{-\psi} \bar{h}_{i j}
$$

where $\left(\bar{h}_{i j}\right)$ is the second fundamental form of the hypersurfaces $\left\{x^{0}=\right.$ const $\}$. 
An easy calculation shows

$$
\bar{h}_{i j} \mathrm{e}^{-\psi}=-\frac{1}{2} \dot{\sigma}_{i j}-\dot{\psi} \sigma_{i j},
$$

where the dot indicates differentiation with respect to $x^{0}$.

\section{Proof of Theorem 1.3}

Let $N$ be a normalized ARW spacetime and assume that the metric is given by (1.4) such that the future end is described by

$$
a \leq x^{0}<0 .
$$

W.l.o.g. we also suppose

$$
\lim _{x^{0} \rightarrow 0} \psi\left(x^{0}, x\right)=0
$$

Let $\left(\tilde{g}_{\alpha \beta}\right)$ be the conformal metric

$$
\tilde{g}_{\alpha \beta} d x^{\alpha} d x^{\beta}=-\left(d x^{0}\right)^{2}+\sigma_{i j}\left(x^{0}, x\right) d x^{i} d x^{j}
$$

and distinguish the geometric quantities with respect to this metric by a tilde, i.e., $\tilde{R}_{\alpha \beta \gamma \delta}$ is the Riemannian curvature tensor, etc. Then we have

$$
\bar{R}_{\alpha \beta}=\tilde{R}_{\alpha \beta}-(n-1)\left[\tilde{\psi}_{\alpha \beta}-\tilde{\psi}_{\alpha} \tilde{\psi}_{\beta}\right]-\tilde{g}_{\alpha \beta}\left[\Delta \tilde{\psi}+(n-1)\|D \tilde{\psi}\|^{2}\right],
$$

and

$$
\bar{R}=\mathrm{e}^{-2 \tilde{\psi}}\left[\tilde{R}-2 n \Delta \tilde{\psi}-n(n-1)\|D \tilde{\psi}\|^{2}\right],
$$

where the covariant derivatives of $\tilde{\psi}$ are taken with respect to the metric $\left(\tilde{g}_{\alpha \beta}\right)$.

The Einstein tensor in $N$ is defined by

$$
G_{\alpha \beta}=\bar{R}_{\alpha \beta}-\frac{1}{2} \bar{R} \bar{g}_{\alpha \beta}
$$

It is divergent free, i.e.,

$$
G_{\beta ; \alpha}^{\alpha}=0
$$

Let $\left(\eta_{\alpha}\right)=\mathrm{e}^{\tilde{\psi}}(-1,0, \ldots, 0)$ be the covariant vector field that represents the future directed normal of the slices $\left\{x^{0}=\right.$ const $\}$ and let $\Omega \subset N$ be an open subset bounded by two space-like hypersurfaces $M_{1}$ and $M_{2}$, where 
$M_{2}$ should lie in the future of $M_{1}$. Applying Gauß' divergence theorem, we obtain

$$
\begin{aligned}
0= & \int_{\Omega} G_{; \alpha}^{\alpha \beta} \eta_{\beta} \mathrm{e}^{\omega f} \mathrm{e}^{\psi} \\
= & -\int_{\Omega} G^{\alpha \beta} \eta_{\beta ; \alpha} \mathrm{e}^{\omega f} \mathrm{e}^{\psi}-\int_{\Omega} G^{\alpha \beta} \eta_{\beta}\left[\omega f_{\alpha}+\psi_{\alpha}\right] \mathrm{e}^{\omega f} \mathrm{e}^{\psi} \\
& -\left(\int_{M_{1}} G_{\alpha \beta} \nu^{\alpha} \eta^{\beta} \mathrm{e}^{\omega f} \mathrm{e}^{\psi}+\int_{M_{2}} G_{\alpha \beta} \nu^{\alpha} \eta^{\beta} \mathrm{e}^{\omega f} \mathrm{e}^{\psi}\right),
\end{aligned}
$$

where the normals $\nu$ of the hypersurfaces $M_{i}$ are supposed to point outward of $\Omega$, i.e., in the case of $M_{1}, \nu$ is past directed, and in the case of $M_{2}, \nu$ is future directed. Note the minus sign in front of the boundary integrals, which is due to the sign of $\langle\nu, \nu\rangle=-1$.

The covariant derivatives of $\left(\eta_{\alpha}\right),\left(\eta_{\alpha ; \beta}\right)$, satisfy

$$
\eta_{0 ; \beta}=0, \quad \eta_{i ; 0}=\bar{\Gamma}_{i 0}^{0} \mathrm{e}^{\tilde{\psi}}=\psi_{, i} \mathrm{e}^{\tilde{\psi}}
$$

where $\psi_{, i}=\partial \psi / \partial x^{i}$, and

$$
\eta_{i ; j}=\bar{\Gamma}_{i j}^{0} \mathrm{e}^{\tilde{\psi}}=-\bar{h}_{i j}
$$

where $\left(\bar{h}_{i j}\right)$ is the second fundamental form of the slices $\left\{x^{0}=\right.$ const $\}$.

Hence we deduce from (3.8)

$$
\begin{aligned}
& \int_{M_{1}} G_{\alpha \beta} \nu^{\alpha} \eta^{\beta} \mathrm{e}^{\omega f} \mathrm{e}^{\psi}+\int_{M_{2}} G_{\alpha \beta} \nu^{\alpha} \eta^{\beta} \mathrm{e}^{\omega f} \mathrm{e}^{\psi} \\
= & \int_{\Omega} G^{i j} \bar{h}_{i j} \mathrm{e}^{\omega f} \mathrm{e}^{\psi}+\int_{\Omega} G^{00}\left[\omega f^{\prime}+\psi^{\prime}\right] \mathrm{e}^{\omega f} \mathrm{e}^{\tilde{\psi}} \mathrm{e}^{\psi} \\
= & \int_{\Omega} n(n-1)\left[f^{\prime \prime}+\tilde{\gamma}\left|f^{\prime}\right|^{2}\right] f^{\prime} \mathrm{e}^{(\omega-3) f} \\
& +\int_{\Omega} c \mathrm{e}^{(\omega-3) f}
\end{aligned}
$$

where the symbol $c$ represents terms that can be estimated by

$$
|c| \leq c_{0}\left(1+\left|f^{\prime}\right|+\epsilon\left|f^{\prime}\right|^{2}\right) \quad \text { and } \quad \lim _{x^{0} \rightarrow 0} \epsilon=0 .
$$

To derive the second equality in (3.11) we used the relations (3.3), (3.4) as well as the assumption that the metrics $\sigma_{i j}(\tau, \cdot)$ converge in $C^{\infty}$ to $\bar{\sigma}_{i j}$. 
The volume element in $\Omega$ is of the form

$$
\mathrm{e}^{(n+1) f} \mathrm{e}^{(n+1) \psi} \sqrt{\operatorname{det}\left(\sigma_{i j}\right)} d x d x^{0} .
$$

Thus the right-hand side of (3.11) vanishes if the hypersurfaces $M_{i}$ approach the singularity, in view of (1.10) and (1.11).

Now, in (3.11) let us choose the hypersurfaces $M_{i}$ to be slices $\left\{x^{0}=\right.$ const\}, then the left-hand side is equal to

$$
\int_{M_{2}} G_{\alpha \beta} \nu^{\alpha} \nu^{\beta} \mathrm{e}^{\omega f} \mathrm{e}^{\psi}-\int_{M_{1}} G_{\alpha \beta} \nu^{\alpha} \nu^{\beta} \mathrm{e}^{\omega f} \mathrm{e}^{\psi}
$$

and we conclude that

$$
\lim _{\tau \rightarrow 0} \int_{M_{\tau}} G_{\alpha \beta} \nu^{\alpha} \nu^{\beta} \mathrm{e}^{\omega f} \mathrm{e}^{\psi}
$$

exists, where $M_{\tau}=\left\{x^{0}=\tau\right\}$, and the limit is equal to

$$
\frac{1}{2} n(n-1) \lim _{\tau \rightarrow 0}\left|f^{\prime}\right|^{2} \mathrm{e}^{(n+\omega-2) f} \int_{\mathcal{S}_{0}} \sqrt{\operatorname{det}\left(\bar{\sigma}_{i j}\right)}=\frac{1}{2} n(n-1) m\left|S^{n}\right| .
$$

From the above considerations, we immediately deduce that the preceding limit is equal to

$$
\lim \int_{M_{k}} G_{\alpha \beta} \nu^{\alpha} \nu^{\beta} \mathrm{e}^{\omega f} \mathrm{e}^{\psi}
$$

where $M_{k}=$ graph $u_{k}$ are arbitrary space-like hypersurfaces, written as graphs over $\mathcal{S}_{0}$, such that

$$
\lim u_{k}=0 \quad \text { and } \quad \lim \left|D u_{k}\right|=0
$$

Hence, we may use the leaves $M(t)$ of an IMCF

$$
\dot{x}=-H^{-1} \nu
$$

with initial hypersurface $M_{0}, H_{\left.\right|_{M_{0}}}>0$, to define the mass, since the flow hypersurfaces $M(t)$ run straight into the singularity and satisfy

$$
|u|_{m} \leq c_{m} \mathrm{e}^{-\gamma t} \quad \forall m \in \mathbb{N},
$$

where $\gamma=\frac{1}{n} \tilde{\gamma}$, cf. [3, Lemma 7.1]. 
Using the Gauß equation

$$
R=-\left[H^{2}-|A|^{2}\right]+2 G_{\alpha \beta} \nu^{\alpha} \nu^{\beta}
$$

we can rewrite

$$
\begin{aligned}
\int_{M} G_{\alpha \beta} \nu^{\alpha} \nu^{\beta} \mathrm{e}^{\omega f} \mathrm{e}^{\psi}= & \frac{n-1}{2 n} \int_{M} H^{2} \mathrm{e}^{\omega f} \mathrm{e}^{\psi} \\
& +\frac{1}{2} \int_{M}\left(R-\left[|A|^{2}-\frac{1}{n} H^{2}\right]\right) \mathrm{e}^{\omega f} \mathrm{e}^{\psi}
\end{aligned}
$$

to conclude that

$$
\lim \int_{M} G_{\alpha \beta} \nu^{\alpha} \nu^{\beta} \mathrm{e}^{\omega f} \mathrm{e}^{\psi}=\lim \frac{n-1}{2 n} \int_{M} H^{2} \mathrm{e}^{\omega f} \mathrm{e}^{\psi}
$$

for those hypersurfaces for which the second integral on the right-hand side of (3.22) tends to zero if the singularity is approached.

This is the case for the coordinate slices $\left\{x^{0}=\right.$ const $\}$ as well as for the leaves of an IMCF.

Lemma 3.1. Let $M(t)$ be a solution of the evolution equation (3.19), then

$$
\lim _{t \rightarrow \infty} \int_{M(t)}\left(R-\left[|A|^{2}-\frac{1}{n} H^{2}\right]\right) \mathrm{e}^{\omega f} \mathrm{e}^{\psi}=0 .
$$

Proof.

(i) Let us first estimate the scalar curvature. We have

$$
\bar{g}_{\alpha \beta}=\mathrm{e}^{2 \tilde{\psi}} \tilde{g}_{\alpha \beta},
$$

where $\left(\tilde{g}_{\alpha \beta}\right)$ is the metric in $(3.3)$. Denote by $\tilde{h}_{i j}, \tilde{g}_{i j}$, etc. the geometric quantities of hypersurfaces when the metric of the ambient space is $\left(\tilde{g}_{\alpha \beta}\right)$, then

$$
\begin{aligned}
\mathrm{e}^{\tilde{\psi}} h_{i}^{j} & =\tilde{h}_{i}^{j}+\tilde{\psi}_{\alpha} \tilde{\nu}^{\alpha} \delta_{i}^{j} \\
g_{i j} & =\mathrm{e}^{2 \tilde{\psi}} \tilde{g}_{i j}=\mathrm{e}^{2 \tilde{\psi}}\left(-u_{i} u_{j}+\sigma_{i j}(u, x) d x^{i} d x^{j}\right),
\end{aligned}
$$

and

$$
R=\mathrm{e}^{-2 \tilde{\psi}}\left(\tilde{R}-2(n-1) \Delta \tilde{\psi}-(n-1)(n-2)\|D \tilde{\psi}\|^{2}\right),
$$

where the covariant derivatives of $\tilde{\psi}(u, x)$ are taken with respect to $\tilde{g}_{i j}$.

Now, $\tilde{R}$ is bounded and the covariant derivatives of $\tilde{\psi}$ are bounded as well, cf. [3, Section 6], hence

$$
\int_{M}|R| \mathrm{e}^{\omega f} \mathrm{e}^{\psi} \leq c \int_{\mathcal{S}_{0}} \mathrm{e}^{(n+\omega-2) f} \mathrm{e}^{(n+1) \psi} \sqrt{\operatorname{det}\left(\sigma_{i j}\right)} \rightarrow 0 .
$$


(ii) To estimate the second fundamental form, we use (3.26) to obtain

$$
\mathrm{e}^{2 \tilde{\psi}}\left(|A|^{2}-\frac{1}{n} H^{2}\right)=|\tilde{A}|^{2}-\frac{1}{n} \tilde{H}^{2}
$$

and thus

$$
\begin{aligned}
& \int_{M}\left(|A|^{2}-\frac{1}{n} H^{2}\right) \mathrm{e}^{\omega f} \mathrm{e}^{\psi} \\
& =\int_{\mathcal{S}_{0}}\left(|\tilde{A}|^{2}-\frac{1}{n} \tilde{H}^{2}\right) \mathrm{e}^{(n+\omega-2) f} \mathrm{e}^{(n-1) \psi} \sqrt{\operatorname{det}\left(\sigma_{i j}\right)} \rightarrow 0 .
\end{aligned}
$$

The final part of Theorem 1.3 is proved in the next section.

\section{Uniqueness of the mass}

The mass of a normalized ARW space does not depend on the particular time function. For a proof of this claim, we shall once again employ the leaves of an IMCF.

Let $\tilde{x}^{0}$ be a second time function that provides a normalized representation of $N$ as an ARW space

$$
d \bar{s}^{2}=\mathrm{e}^{2(\tilde{f}+\tilde{\psi})}\left(-\left(d \tilde{x}^{0}\right)^{2}+\tilde{\sigma}_{i j} d x^{i} d x^{j}\right)
$$

such that

$$
\lim \left|\tilde{f}^{\prime}\right|^{2} \mathrm{e}^{(n+\tilde{\omega}-2) \tilde{f}}=\tilde{m}>0 .
$$

Let $M(t)$ be a solution of the evolution problem (3.19) which are written as $M(t)=$ graph $u$ in the original coordinate system and as $M(t)=$ graph $\tilde{u}$ in the new system. In both cases, we may assume that the hypersurfaces are graphs over $\mathcal{S}_{0}$, since it is unnecessary that $\mathcal{S}_{0}$ is a level hypersurface for a time function. The estimates (3.20) are satisfied by $u$ as well as $\tilde{u}$.

There are many invariants that could be used to compare $f$ and $\tilde{f}$. Let us consider $G_{\alpha \beta} \nu^{\alpha} \nu^{\beta}$ evaluated at $M(t)$. Arguing as in the preceding section, we deduce

$$
1=\lim _{t \rightarrow \infty} \frac{\left|f^{\prime}\right|^{2} \mathrm{e}^{(n+\omega-2) f}}{\left|\tilde{f}^{\prime}\right|^{2} \mathrm{e}^{(n+\omega-2) \tilde{f}}} \frac{\mathrm{e}^{(n+\tilde{\omega}) \tilde{f}}}{\mathrm{e}^{(n+\omega) f}},
$$

where the arguments of $f, f^{\prime}$ resp. $\tilde{f}, \tilde{f}^{\prime}$ are $u$ resp. $\tilde{u}$. 
Hence we conclude

$$
\lim \frac{\mathrm{e}^{(n+\tilde{\omega}) \tilde{f}}}{\mathrm{e}^{(n+\omega) f}}=\frac{\tilde{m}}{m}
$$

in view of (1.10) and (4.2).

Now we observe that

$$
\frac{d}{d t} f(u)=f^{\prime} \dot{u}=\frac{\tilde{v} f^{\prime}}{\tilde{H}-n \tilde{v} f^{\prime}+\psi_{\alpha} \tilde{\nu}^{\alpha}},
$$

where $\tilde{H}$ is the mean curvature with respect to the conformal metric in (3.3) and $\tilde{v}=v^{-1}$. Hence we obtain

$$
\lim _{t \rightarrow \infty} \frac{d}{d t} f(u)=-\frac{1}{n}
$$

The same result is of course valid for $(d / d t) \tilde{f}(\tilde{u})$, where one should note the ambiguous usage of the tilde.

Combining (4.4), (4.6), and de L'Hospital's rule, we infer that $\omega=\tilde{\omega}$.

To prove

$$
\lim \int_{M(t)} G_{\alpha \beta} \nu^{\alpha} \nu^{\beta} \mathrm{e}^{\omega f} \mathrm{e}^{\psi}=\lim \int_{M(t)} G_{\alpha \beta} \nu^{\alpha} \nu^{\beta} \mathrm{e}^{\omega \tilde{f}} \mathrm{e}^{\tilde{\psi}}
$$

it suffices to show that $m=\tilde{m}$, or equivalently,

$$
\lim \frac{\mathrm{e}^{\tilde{f}}}{\mathrm{e}^{f}}=1 .
$$

Since $\omega=\tilde{\omega}$, relation (4.4) implies

$$
\lim \frac{\mathrm{e}^{\tilde{f}}}{\mathrm{e}^{f}}=c=\text { const. }
$$

Let $\left(\xi^{i}\right)$ be local coordinates for $M(t)$ and $x=x(\xi)$ be a local embedding, then

is the induced metric. Let

$$
g_{i j}=\left\langle x_{i}, x_{j}\right\rangle
$$

$$
\tilde{g}_{i j}=\mathrm{e}^{-2(f+\psi)} g_{i j}
$$

be the conformal metric, then

$$
\int_{\mathcal{S}_{0}} \sqrt{\operatorname{det}\left(\tilde{g}_{i j}\right)}=\int_{\mathcal{S}_{0}} v \sqrt{\operatorname{det}\left(\sigma_{i j}(u, x)\right.}
$$

where

$$
v^{2}=1-|D u|^{2}=1-\sigma^{i j} u_{i} u_{j}
$$


and hence

$$
\lim \int_{\mathcal{S}_{0}} v \sqrt{\operatorname{det}\left(\sigma_{i j}(u, x)\right)}=\left|S^{n}\right|
$$

due to our normalization assumption.

On the other hand, if we express the right-hand side of (4.11) with respect to the second coordinate system, then we obtain

$$
\left|S^{n}\right|=\lim \int_{\mathcal{S}_{0}} \mathrm{e}^{n(\tilde{f}+\tilde{\psi})} \mathrm{e}^{-n(f+\psi)} v \sqrt{\operatorname{det}\left(\tilde{\sigma}_{i j}(\tilde{u}, x)\right)}=c^{n}\left|S^{n}\right|,
$$

hence $c=1$.

\section{A variant of the Penrose inequality}

Let $M_{\tau}=\left\{x^{0}=\tau\right\}$ be coordinate slices and suppose that the integrals

$$
\int_{M_{\tau}} G_{\alpha \beta} \nu^{\alpha} \nu^{\beta} \mathrm{e}^{\omega f} \mathrm{e}^{\psi}
$$

would increase monotonically with respect to $\tau$, then

$$
\lim _{\tau \rightarrow 0} \int_{M_{\tau}} G_{\alpha \beta} \nu^{\alpha} \nu^{\beta} \mathrm{e}^{\omega f} \mathrm{e}^{\psi} \geq \int_{M_{\bar{\tau}}} G_{\alpha \beta} \nu^{\alpha} \nu^{\beta} \mathrm{e}^{\omega f} \mathrm{e}^{\psi}
$$

If $M_{\bar{\tau}}$ would be totally geodesic, then

$$
\int_{M_{\bar{\tau}}} G_{\alpha \beta} \nu^{\alpha} \nu^{\beta} \mathrm{e}^{\omega f} \mathrm{e}^{\psi}=\frac{1}{2} \int_{M_{\bar{\tau}}} R \mathrm{e}^{\omega f} \mathrm{e}^{\psi}
$$

To prove the monotonicity of the integrals in (5.1), let us look at the relation (3.11). The monotonicity of the integrals is equivalent to the nonnegativity of the right-hand side of (3.11). This will be the case for highly symmetrical spacetimes, as we shall see in the next section. For general ARW spacetimes however, non-negativity of the right-hand side of (3.11) could only be derived under the assumptions $\omega=0, \psi=0$, and, furthermore, that the slices $M_{\tau}$ are convex, i.e., $\bar{h}_{i j} \geq 0$, and the spatial part of the Einstein tensor positive semi-definite, i.e., $G^{i j} \geq 0$.

Notice that $f^{\prime}$ is always negative and that, under physical assumptions, $G^{00} \geq 0$ and also that the signs of the spatial part $\left(G^{i j}\right)$ and of $\omega$ are the 
same, as can be derived from the Einstein equations

$$
G_{\alpha \beta}=\kappa T_{\alpha \beta},
$$

if the stress energy tensor is supposed to be asymptotically that of a perfect fluid with an equation of state

$$
p=\frac{\omega}{n} \rho .
$$

\section{$6 \quad$ An example}

Let $\hat{N}$ be the $\mathrm{S}_{-\mathrm{AdS}_{(n+2)}}$ spacetime with metric

$$
d \hat{s}^{2}=-h d t^{2}+h^{-1} d r^{2}+r^{2} \sigma_{i j} d x^{i} d x^{j},
$$

where

$$
h=1-\frac{2}{n(n+1)} \Lambda r^{2}-m r^{-(n-1)}
$$

with constants $\Lambda \leq 0$ and $m>0 ;\left(\sigma_{i j}\right)$ is the metric of $S^{n}$.

In $r=0$, there is a black hole singularity, the event horizon is in $r=$ $r_{0}$, such that $h\left(r_{0}\right)=0$, and the black hole region is given by $\{h<0\}=$ $\left\{0<r<r_{0}\right\}$.

In the black hole region, $t$ is a spatial coordinate and $r$ the time coordinate. Set

$$
\tilde{h}=-h
$$

and consider in the black hole region, the brane

$$
N=\left\{t=\text { const, } 0<r<r_{0}\right\} .
$$

The induced metric $\left(\bar{g}_{\alpha \beta}\right)$ is

$$
\begin{aligned}
d \bar{s}^{2} & =-\tilde{h}^{-1} d r^{2}+r^{2} \sigma_{i j} d x^{i} d x^{j} \\
& =r^{2}\left(-r^{-2} \tilde{h}^{-1} d r^{2}+\sigma_{i j} d x^{i} d x^{j}\right)
\end{aligned}
$$

Define

and $x^{0}$ by

$$
f=\log r
$$

$$
\begin{aligned}
& d x^{0}=-r^{-1} \tilde{h}^{-1 / 2} d r, \\
& \text { i.e., } \quad x^{0}=-\int_{0}^{r} s^{-1} \tilde{h}^{-1 / 2} d s .
\end{aligned}
$$

$x^{0}$ is then a future directed time function and the (induced) singularity lies in $x^{0}=0$. 
In these coordinates, the metric has the form

$$
d \bar{s}^{2}=\mathrm{e}^{2 f}\left(-\left(d x^{0}\right)^{2}+\sigma_{i j} d x^{i} d x^{j}\right) .
$$

Let a prime denote differentiation with respect to $x^{0}$ and a dot with respect to $r$, then

and

$$
f^{\prime}=r^{-1} \frac{d r}{d x^{0}}=-\tilde{h}^{1 / 2}
$$

$$
f^{\prime \prime}=\frac{1}{2} r \dot{\tilde{h}}=-\frac{1}{2} m(n-1) r^{-(n-1)}+\frac{2}{n(n+1)} \Lambda .
$$

Set $\omega=1$ so that

$$
\tilde{\gamma}=\frac{1}{2}(n+\omega-2)=\frac{1}{2}(n-1)
$$

then

and

$$
f^{\prime \prime}+\tilde{\gamma}\left|f^{\prime}\right|^{2}=\frac{1}{n} \Lambda r^{2}-\frac{1}{2}(n-1)
$$

$$
\left|f^{\prime}\right|^{2} \mathrm{e}^{2 \tilde{\gamma} f}=\left|f^{\prime}\right|^{2} r^{(n-1)}=\tilde{h} r^{(n-1)}=m+\frac{2}{n(n+1)} \Lambda r^{(n+1)}-r^{(n-1)} .
$$

Let $M_{r}$ be the coordinate slices $\left\{x^{0}=\right.$ const $\}$, then the past directed normal is $\nu=\left(\nu^{\alpha}\right)=\mathrm{e}^{-f}(-1, \ldots, 0)$,

$$
G_{\alpha \beta} \nu^{\alpha} \nu^{\beta} \mathrm{e}^{2 f}=\frac{1}{2} n(n-1)\left|f^{\prime}\right|^{2}+\frac{1}{2} n(n-1)=\frac{1}{2} n(n-1)(\tilde{h}+1),
$$

and

$$
\int_{M_{r}} G_{\alpha \beta} \nu^{\alpha} \nu^{\beta} \mathrm{e}^{f}=\frac{1}{2} n(n-1) \int_{S^{n}}(\tilde{h}+1) r^{(n-1)},
$$

hence

$$
\lim _{r \rightarrow 0} \int_{M_{r}} G_{\alpha \beta} \nu^{\alpha} \nu^{\beta} \mathrm{e}^{f}=\frac{1}{2} n(n-1)\left|S^{n}\right| m .
$$

The convergence is monotone increasing in $x^{0}$.

Note that the value $\omega=1$ corresponds to a radiation dominated universe if the stress energy tensor is asymptotically equal to that of a perfect fluid and the equation of state is

$$
p=\frac{\omega}{n} \rho
$$

\section{Acknowledgment}

This work has been supported by the Deutsche Forschungsgemeinschaft. 


\section{References}

[1] R. Bartnik, The mass of an asymptotically flat manifold, Comm. Pur. Appl. Math. 39(5) (1986), 661-693, MR 88b:58144 .

[2] C. Gerhardt, Hypersurfaces of prescribed curvature in Lorentzian manifolds, Indiana U. Math. J. 49 (2000), 1125-1153, pdf file.

[3] C. Gerhardt, The inverse mean curvature flow in ARW spaces transition from big crunch to big bang, 2004, arXiv:math.DG/0403485, 39 pages.

[4] C. Gerhardt, The inverse mean curvature flow in cosmological spacetimes, 2004, arXiv:math.DG/0403097, 24 pages.

[5] G. Huisken and T. Ilmanen, The inverse mean curvature flow and the Riemannian Penrose inequality, J. Diff. Geom. 59(3) (2001), 353-437.

[6] R. Schoen and S. T. Yau, Positivity of the total mass of a general spacetime, Phys. Rev. Lett. 43(20) (1979), 1457-1459, MR 81c:58024 .

[7] R. Schoen and S. T. Yau, Proof of the positive mass theorem. II, Comm. Math. Phys. 79(2) (1981), 231-260, MR 83i:83045. 